\title{
Study on the anticancer biological mechanism of Resveratrol
}

\author{
DU Juan ${ }^{1, \mathrm{a}}$, YANG Dongsheng ${ }^{1}$, HU Wenzhong ${ }^{1}$, MENG Fanxin ${ }^{1}$, LIN Zhongping ${ }^{2}$ \\ ${ }^{1}$ College of Pharmacy and Food Science, Zhuhai College of Jilin University, Zhuhai 519041, China. \\ ${ }^{2}$ School of Life Sciences, Peking University, Beijing, 100871, China
}

\begin{abstract}
Trans - 3, 4', 5-3 hydroxy 2 styrene (resveratrol) is a kind of naturally occurring polyphenols phytoalexin. Resveratrol has significant anti-cancer activity, mainly exists in grapes, berries, and peanuts, has anti-aging, protect the heart, antioxidant, anti- proliferation, promote apoptosis and immune regulation. Resveratrol has been widely concerned in the treatment of cancer and autoimmune diseases resveratrol. Resveratrol synthase (RS) is a key enzyme in the synthesis of resveratrol synthase. In this study, RS containing genes were placed under the control of fruit-specific promoter RJ39 to transform tomatoes by transgenic method. The extraction of fruits containing RS genes showed an obvious absorption peak on the HPLC chromatographic map, and it also had an obvious inhibitory effect on the growth of Hela cells.
\end{abstract}

\section{Introduction}

Resveratrol (RES) was first extracted from the root of Veratrum puberulum Loes. Up to now, Resveratrol has been obtained from more than 70 kinds of plants, such as grape, peanut and Polygonum cuspate. In recent years, studies have shown that resveratrol has a variety of biological activities, including anti-oxidation ${ }^{[1]}$, antiaging $^{[2]}, \quad$ anti-inflammatory ${ }^{[3]}, \quad$ anti-cancer ${ }^{[4]}$, cardiovascular protection $^{[5]}$, neuroprotection ${ }^{[6]}$, immune regulation $^{[7]}$, treatment of obesity, diabetes, osteoporosis ${ }^{[8]}$, antiviral ${ }^{[9]}$ and as a therapeutic drug for viral infection ${ }^{[10]}$. Resveratrol can regulate the proliferation of human prostate cancer cells in vitro. Resveratrol can inhibit the proliferation of PC-3 cells, block pc-3 cells in G0/G1 phase, increase the expression of Bax protein and decrease the expression of Survivin protein, and directly activate Caspase- 3 and inhibit the proliferation of PC-3 cells $^{[11]}$. Resveratrol also plays an important role in $\mathrm{PCa}$ cell proliferation and migration. An E3 ligase, namely TNF - receptor-related factor 6 (TRAF6), is a key mediator for resveratrol to inhibit $\mathrm{PCa}$ cell growth and proliferation, and resveratrol can be used as a target for lysosomal degradation to inhibit $\mathrm{PCa}$ cell proliferation and migration ${ }^{[12]}$.RES can slow down the growth of PC3 cells by interfering with glucose fermentation and promoting respiration, and effectively inhibit the growth of PC3 cells under hypoxic conditions, thus avoiding dependence on oxidative phosphorylation ${ }^{[13]}$.The ability of RSV to directly target PD-L1 interferes with its stability and transport, and ultimately prevents it from targeting the plasma membrane of cancer cells ${ }^{[14]}$. Resveratrol has the ability to down regulate viral oncogene E6, and can be used as a potential anti-HPV E6 + tumor drug for cervical cancer and ovarian cancer caused by human papillomavirus (HPV) ${ }^{[15,16]}$. RES regulates the level of Galectin-3 (GAL-3), which is an anti-apoptotic lectin that is highly overexpressed in ovarian cancer cells. RES induces cell death in a dose dependent manner, inhibits cell migration and invasion, and down-regulates bcl-2 levels, increasing the degradation of lysed Caspase- 3 and Gal-3 proteins (but not mRNA) ${ }^{[17]}$.Simultaneous administration of calcitonin and curcumin or resveratrol has a synergistic promoting effect on anticancer effect of human breast tumor cells in vitro and in vivo and inhibits the formation of tumor neovascularization ${ }^{[18]}$. Res is not only a kind of plant antitoxin, but also has an obvious health care effect on human body ${ }^{[19]}$. However, resveratrol content in nature is relatively low, so the use of transgenic technology to improve the content of resveratrol in edible plants has become a new research hotspot. In this study, the transformation of resveratrol synthase RS gene into tomatoes was expected to improve the nutritional quality of tomatoes and to increase the content of resveratrol in tomato fruits.

\section{Materials and methods}

The specific promoter RJ39 was cloned from of strawberry. The RS gene was cloned from of Clonagalus citrine, 1179 bp encoding 392 amino acids. The herbicide resistance gene was selected as the marker gene to construct the plant expression vector Pcambia 3300-RJ39RS-T, which was preserved by the State Key Laboratory of Plant Genetic engineering and Protein Engineering, School of Life Sciences, Peking University.The constructed expression vector was transferred into agrobacterium tumefaciens LBA4404 with high conversion efficiency, and was used to transform tomatoes. 


\section{3 results}

Expression vector pCAMBIA3300-RJ39-RS-T See

Figure 1 for the results.

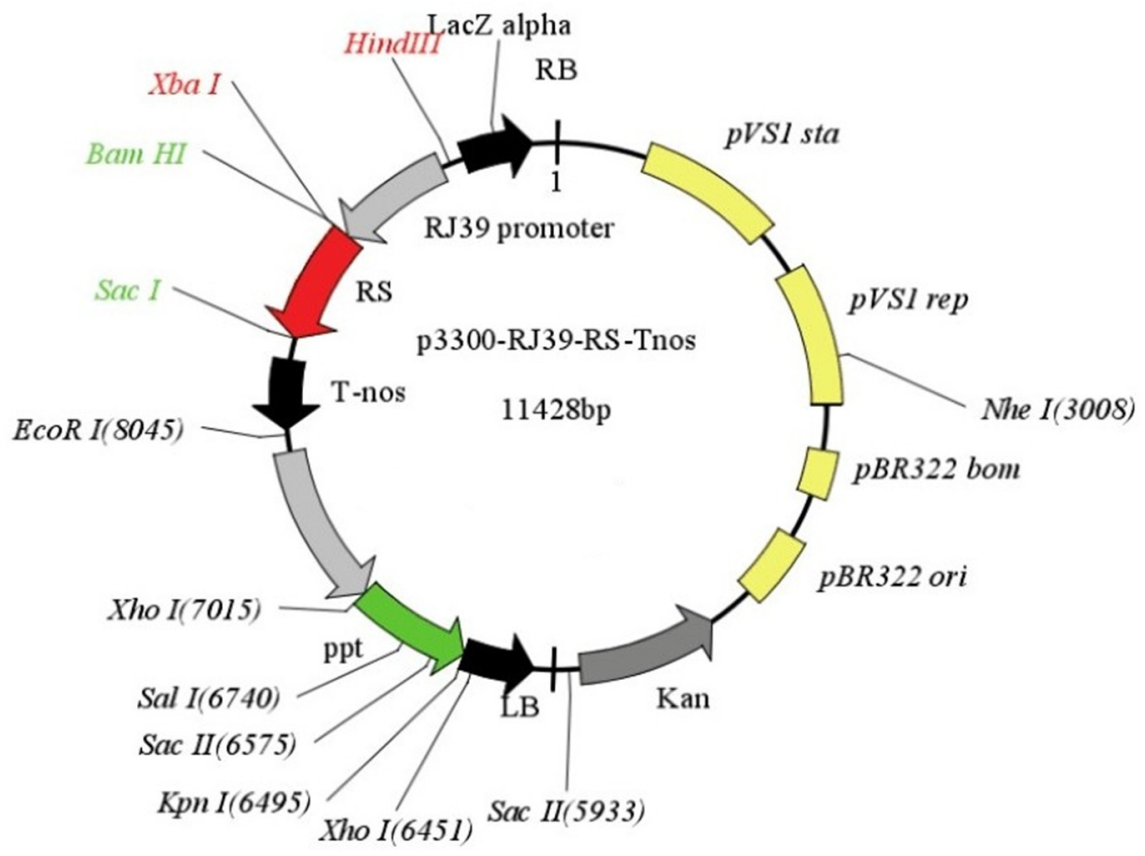

Fig1. Expression vector pCAMBIA3300-RJ39-RS-T

The PCR and Southern blotting showed that the RS gene had been transformed into plant. See Figure 2 for the results.

\section{$\begin{array}{lllllllllllllllll}M & 1 & 2 & 3 & 4 & 5 & 6 & 7 & 8 & 9 & 10 & 11 & 12 & 13 & 14 & 15 & 16\end{array}$}

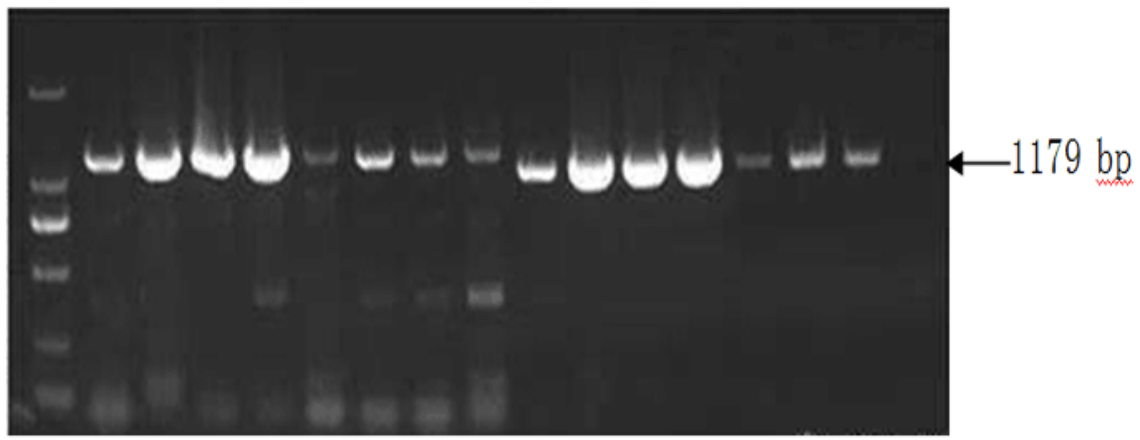

Fig2. Figure 2 the PCR showed that the RS gene had been transformed into plant.M:Marker;1-15 positive control; 16: negative control

The results indicated that the transferred exogenous RS gene could be normally transcribed and translated into resveratrol synthase capable of normal function, and catalyzed the production of resveratrol by substrates in allogenic plants. The high resolution of HPLC enables more accurate analysis of these compounds, as shown in Figure 3: These compounds show different retention times on the HPLC. 


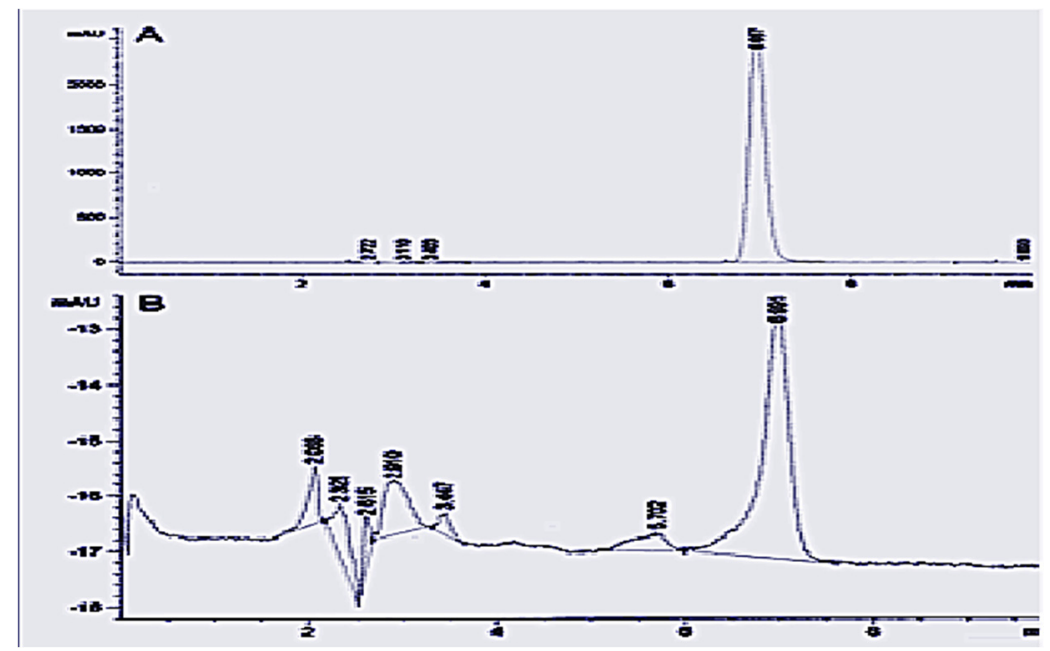

Fig3. HPLC analysis of trans-resveratrol and transgenic plant extracts A: Trans-resveratrol standard B: Genetically modified plant extracts

In order to detect the function of resveratrol extracted from transresveratrol synthase tomato fruit, the effect of resveratrol on HELA cell growth was studied.The effect of resveratrol extracted from transgenic tomato fruits on HELA cell growth was observed by preparation of $200 \mathrm{M}$ DMSO solution (FIG. 4).Pictures were taken 24 hours after induction. Figure A shows the growth of HELA cells in DMEM medium.Figure B shows HELA cells growing in DMEM medium containing resveratrol extracted from
$200 \mathrm{M}$ transresveratrol synthase tomato fruit.The cells shown in Figure A were findle-shaped and adherent to the wall, which indicated that the growth state of the cells was normal. The cells shown in figure B began to shrink and deform, and were suspended in the culture. This result preliminarily indicated that HELA cells began to shrink and die.It was also demonstrated that resveratrol in the fruit of the tomato with resveratrol synthase had the same function as standard products.
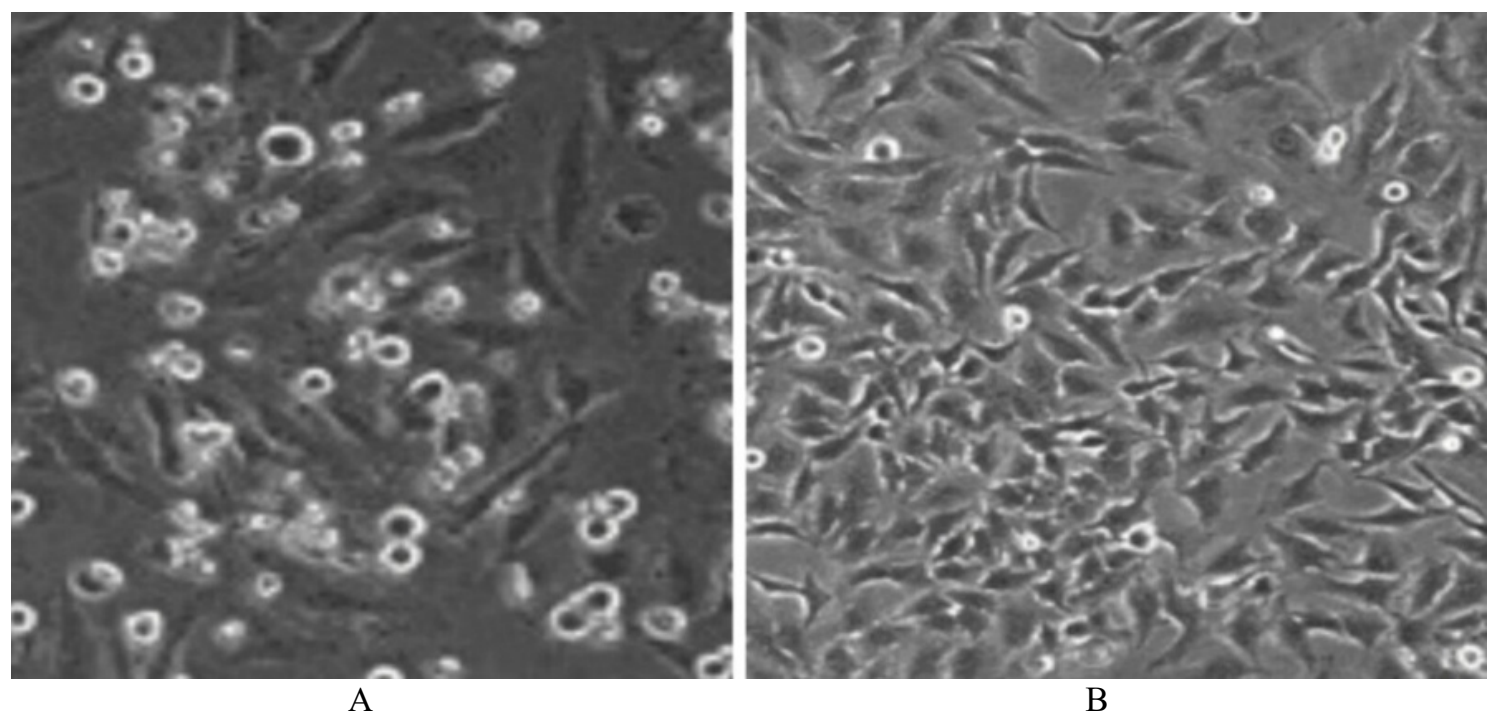

Fig4. Effects of resveratrol extracted from transgenic lettuce on HELA cell growth. A: DMEM medium. B: DMEM medium for HELA cells extracted from RS tomato fruit containing $200 \mathrm{M}$.

\section{Conclusion}

Resveratrol Synthase (RS) is a key enzyme in the synthesis of Resveratrol.Studies have reported that RS gene has been transformed and expressed in a variety of plants and microorganisms, and plays a biological role in plant metabolism and regulation.In this study, the RS fulllength sequence (1.179 KB) was cloned from Parthenocissus Henryana, a grape plant. 392 amino acids were encoded.Use fruit specific promoters RJ39 construction of plant expression vector pCAMBIA3300 RJ39 - RS - T.RS gene was transferred into strawberry and tomato by agrobacterium transformation. Fruit extract of strawberry and tomatoes turn RS gene HPLC chromatographic analysis, to demonstrate the synthesis of resveratrol in transgenic plants.Extraction of resveratrol in transgenic fruits, and the resveratrol inhibits the growth of the cervical cancer cell line Hela experiment, colleagues expressing activity of resveratrol were analyzed.Increasing the content of resveratrol in edible plants by transgenic technology has become a new research hotspot.The conversion of resveratrol synthase 
RS gene into the fruits of allogeneic plants is expected to improve the nutritional quality of the fruits, and try to increase the content of resveratrol in the fruits, so as to achieve the effect of nutrition, health care and disease prevention.

\section{Acknowledgement}

Special thanks to all the staff at The Linzhongping Laboratory of Peking University.Thanks to Zhuhai College of Jilin University for the project funding. We are deeply grateful to Linzhongping, HU Wenzhong , YANG Dongsheng, MENG Fanxin for his support and work in this study.

Fund project: Zhuhai College of Jilin University Innovation Ability Cultivation Project Fund (2019XJCQ005)

\section{About the author:}

Du Juan, female, doctor, associate Researcher research direction: Biochemistry and molecular biology Tel : 15812640083 E-mail: 84453594@qq.com

\section{REFERENCES}

1. BRENJIAN S, MOINI A, YAMINI N, et al. Resveratrol treatment in patients with polycystic ovary syndrome decreased pro-inflammatory and endoplasmic reticulum stress markers[J]. $A m \quad J$ Reprod Immunol, 2020, 83(1): doi: 10.1111/aji.13186.

2. KHAFAGA A F, NORELDIN A E, TAHA A E. The adaptogenic anti-ageing potential of resveratrol against heat stress-mediated liver injury in aged rats: Role of HSP70 and NF-kB signalling[J]. J Therm Biol, 2019, 83: 8-21.

3. ALRAFAS H R, BUSBEE P B, NAGARKATTI M, et al. Resveratrol Downregulates miR-31 to Promote $\mathrm{T}$ Regulatory Cells during Prevention of TNBSInduced Colitis[J]. Mol Nutr Food Res, 2020, 64(1): doi: $10.1002 / \mathrm{mnfr} .201900633$.

4. KHUSBU F Y, ZHOU X, ROY M, et al. Resveratrol induces depletion of TRAF6 and suppresses prostate cancer cell proliferation and migration[J]. Int $J$ Biochem Cell B, 2020, 118: doi: 10.1016/j.biocel.2019.105644.

5. ASADPOUR S, YEGANEH H, KHADEMI F, et al. Resveratrol-loaded polyurethane nanofibrous scaffold: viability of endothelial and smooth muscle cells[J]. Biomed Mater, 2020, 15(1): doi: 10.1088/1748-605X/ab4e23.

6. ABD AZIZ N A W, IEZHITSA I, AGARWAL R, et al. Neuroprotection by trans-resveratrol against collagenase-induced neurological and neurobehavioural deficits in rats involves adenosine A1 receptors[J]. Neurol Res, 2020, 42(3): 189-208.
7. HSU Y J, HO C S, LEE M C, et al. Protective Effects of Resveratrol Supplementation on Contusion Induced Muscle Injury[J]. Int J Med Sci, 2020, 17(1): 53-62.

8. ALI D, CHEN L, KOWAL J M, et al. Resveratrol inhibits adipocyte differentiation and cellular senescence of human bone marrow stromal stem cells $[\mathrm{J}]$. Bone, 2020, 133: doi: 10.1016/j.bone.2020.115252.

9. ZHAO X, TONG W, SONG X, et al. Antiviral Effect of Resveratrol in Piglets Infected with Virulent Pseudorabies Virus[J]. Viruses, 2018, 10(9): doi:10.3390/v10090457.

10. PAEMANEE A, HITAKARUN A, ROYTRAKUL S, et al. Screening of melatonin, alpha-tocopherol, folic acid, acetyl-L-carnitine and resveratrol for antidengue 2 virus activity[J]. BMC Res Notes, 2018, 11(1): doi: 10.1186/s13104-018-3417-3.

11. ATMACA H, BOZKURT E, KISIM A, et al. Comparative analysis of XTT assay and xCELLigence system by measuring cytotoxicity of resveratrol in human cancer cell lines[J]. Turk $J$ Biochem, 2016, 41(6): 413-421.

12. KHUSBU F Y, ZHOU X, ROY M, et al. Resveratrol induces depletion of TRAF6 and suppresses prostate cancer cell proliferation and migration[J]. International Journal of Biochemistry \& Cell Biology, 2020, 118:

13. FONSECA J, MORADI F, MADDALENA LA, et al. Resveratrol integrates metabolic and growth effects in PC3 prostate cancer cells-involvement of prolyl hydroxylase and hypoxia inducible factor-1[J]. Oncol Lett, 2019, 17(1): 697-705.

14. VERDURA S, CUYAS E, CORTADA E, et al. Resveratrol targets PD-L1 glycosylation and dimerization to enhance antitumor T-cell immunity $[\mathrm{J}]$. Aging-Us, 2020, 12(1): 8-34.

15. OMIDIAN K, RAFIEI H, BANDY B. Increased mitochondrial content and function by resveratrol and select flavonoids protects against benzo[a]pyreneinduced bioenergetic dysfunction and ROS generation in a cell model of neoplastic transformation[J]. Free Radic Biol Med, 2020: doi: 10.1016/j.freeradbiomed.2020.01.021.

16. EL-KOTT A F, SHATI A A, AL-KAHTANI M A, et al. The apoptotic effect of resveratrol in ovarian cancer cells is associated with downregulation of galectin-3 and stimulating miR-424-3p transcription[J]. J Food Biochem, 2019: doi: $10.1111 / \mathrm{jfbc} .13072$.

17. EL-KOTT A F, SHATI A A, AL-KAHTANI M A, et al. The apoptotic effect of resveratrol in ovarian cancer cells is associated with downregulation of galectin-3 and stimulating miR-424-3p transcription[J]. Journal of Food Biochemistry, 2019:

18. GARCIA-QUIROZ J, GARCIA-BECERRA R, SANTOS-CUEVAS C, et al. Synergistic Antitumorigenic Activity of Calcitriol with Curcumin or Resveratrol is Mediated by Angiogenesis 
Inhibition in Triple Negative Breast Cancer Xenografts[J]. Cancers, 2019, 11(11): doi: 10.3390/cancers11111739.

19. Zhong J, Liu SJ, Ma SS, et al. Effect of matrix ttachment regions on resveratrol production in tobacco with transgene of stilbene synthase from Parthenocissus henryana. Acta Botan Sin, 2004, 46(8): 948-954 (in Chinese). 